\title{
Serviço público e educação on-line durante a pandemia de Covid-19: um estudo sobre o perfil dos usuários da plataforma Enem Pará
}

Public Service and Online Education During the Covid-19 Pandemic: a study on the profile of users of the Enem Pará platform

Servicio público y educación en línea durante la pandemia Covid-19: un estudio sobre el perfil de los usuarios de la plataforma Enem Pará

Yuri Vidal Santiago de Mendonça ORCID: https://orcid.org/0000-0002-0636-4775 Universidade Federal do Pará, Brasil E-mail: yurisantiago@ufpa.br

Everaldo Marcelo Souza da Costa ORCID: https://orcid.org/0000-0001-5134-4733 Universidade da Amazônia, Brasil E-mail: prof.emsc@gmail.com

Thalita Carvalho Bezerra

ORCID: https://orcid.org/0000-0002-0116-1055 Universidade da Amazônia, Brasil

E-mail: thalitacarvalhobezerra@gmail.com

Igor de Jesus Lobato Pompeu Gammarano ORCID: https://orcid.org/0000-0001-7769-8265 Universidade da Amazônia, Brasil

E-mail: igor.internacionalista@gmail.com

\begin{abstract}
Resumo
A plataforma ENEM PARÁ foi criada durante a pandemia de Covid-19 com o objetivo de democratizar o conhecimento, permitindo que alunos da rede pública de ensino do estado do Pará possam ter acesso a conteúdos preparatórios para o Exame Nacional do Ensino Médio - ENEM. O objetivo deste trabalho é analisar através do acesso e dados da plataforma ENEM PARÁ, o perfil socioeconômico dos usuários dela, visto que através dessa investigação é possível abranger os diferentes públicos que a acessam, podendo resultar numa comunicação mais assertiva no desenvolvimento e compartilhamento de novos conteúdos da plataforma. Os objetivos dessa pesquisa têm caráter exploratório quanto à abordagem do problema e caracteriza-se como quantitativa, com o uso de dados secundários, pois esses foram disponibilizados pelos gerenciadores da plataforma ENEM PARÁ para o desenvolvimento do estudo, e a apresentação dos seus resultados se deu por meio de estatística descritiva e análises cruzadas com o uso do software SPSS, com foco na verificação do perfil dos usuários. Os resultados indicaram que a plataforma tem conseguido atingir seus objetivos, porém a maioria dos alunos da rede pública estadual ainda não acessam o serviço. Este trabalho busca contribuir com as estratégias de comunicação e inclusão digital de mais estudantes paraenses na plataforma ENEM PARÁ, entendendo que esse é um importante serviço público que o governo do estado tem oferecido para aqueles que irão prestar o exame nacional do ensino médio.
\end{abstract}

Palavras-chave: Plataformas de educação on-line; Serviço público; Perfil de usuários; Ensino.

\begin{abstract}
The ENEM PARÁ platform was created during the Covid-19 pandemic with the objective of democratizing knowledge, allowing students from the public education system in the state of Pará to have access to preparatory content for the National High School Exam - ENEM. The objective of this work is to analyze, through the access and data of the ENEM PARÁ platform, the socioeconomic profile of its users, since through this investigation it is possible to cover the different audiences that access it, which can result in a more assertive communication in the development and sharing of new platform contents. The objectives of this research are exploratory in terms of approaching the problem and are characterized as quantitative, with the use of secondary data, as these were made available by the managers of the ENEM PARÁ platform for the development of the study, and the presentation of its results took place through descriptive statistics and cross-analysis using the SPSS software, with a focus on verifying the profile of users. The results indicated that the platform has managed to achieve its goals, but most students in the state public network still do not access the service. This work seeks to contribute to the communication and digital
\end{abstract}


inclusion strategies of more students from Pará on the ENEM PARÁ platform, understanding that this is an important public service that the state government has offered to those who will take the national high school exam.

Keywords: Online education platforms; Public service; Users profile; Teaching.

\begin{abstract}
Resumen
La plataforma ENEM PARÁ fue creada durante la pandemia Covid-19 con el objetivo de democratizar el conocimiento, permitiendo que los estudiantes del sistema de educación pública del estado de Pará tengan acceso a contenidos preparatorios para el Examen Nacional de Bachillerato - ENEM. El objetivo de este trabajo es analizar, a través del acceso y datos de la plataforma ENEM PARÁ, el perfil socioeconómico de sus usuarios, ya que a través de esta investigación es posible cubrir las diferentes audiencias que acceden a él, lo que puede resultar en una respuesta más asertiva. comunicación en el desarrollo y puesta en común de nuevos contenidos de plataforma. Los objetivos de esta investigación son exploratorios en cuanto al abordaje del problema y se caracterizan por ser cuantitativos, con el uso de datos secundarios, ya que estos fueron puestos a disposición por los responsables de la plataforma ENEM PARÁ para el desarrollo del estudio, y la presentación de sus resultados se realizaron mediante estadística descriptiva y análisis cruzado utilizando el software SPSS, con un enfoque en la verificación del perfil de los usuarios. Los resultados indicaron que la plataforma ha logrado alcanzar sus metas, pero la mayoría de los estudiantes de la red pública estatal aún no acceden al servicio. Este trabajo busca contribuir a las estrategias de comunicación e inclusión digital de más estudiantes de Pará en la plataforma ENEM PARÁ, entendiendo que este es un importante servicio público que el gobierno estatal ha brindado a quienes presentarán el examen nacional de bachillerato.
\end{abstract}

Palabras clave: Plataformas de educación en línea; Servicio público; Perfil de usuarios; Enseñanza.

\title{
1. Introdução
}

Atualmente o uso das plataformas on-line tem se sobressaído, visto sua dinâmica de uso, bem como o desenvolvimento, difusão e manutenção de conteúdos e serviços nos mais diversos tipos de cenários, como saúde, alimentação, transporte, hospedagem, venda e educação. Além disso, a utilização dessas plataformas gera ao indivíduo ou instituição, algum tipo de valor, seja ele hedônico, pelo seu aspecto de diversão, prazer e experiência afetiva ou valor utilitário, através do seu benefício prático, funcional e útil (Alexandroni \& Arruda Filho, 2017).

Nesse contexto, o setor educacional desenvolveu e/ou adaptou o uso dessas plataformas, em virtude do cenário pandêmico causado pela Covid-19, tornando frequente sua utilização para o compartilhamento de conteúdo educacionais (PARADA, et al., 2020), com o intuito de oportunizar o acesso à aprendizagem através das tecnologias digitais e de comunicação (TDICS) disponíveis, as quais possibilitam a interação, o desenvolvimento cultural, social e educacional (SOARES, et al., 2020).

Nessa perspectiva, foi desenvolvida no Estado do Pará a plataforma on-line Enem Pará, através da Secretaria de Estado de Ciência, Tecnologia e Educação Tecnológica do Estado (SECTET), com o objetivo de democratizar o conhecimento para os alunos da rede pública, em especial aqueles que prestarão o Exame Nacional do Ensino Médio (ENEM). Nesta plataforma, são disponibilizados de forma gratuita, conteúdos em forma de videoaulas, material para leitura, simulados e exercícios das diversas áreas do conhecimento, produzidos por professores experientes e capacitados, além de pesquisas de satisfação e gamificação de conteúdos como estratégia didática de ensino (Martins, Giraffa \& Rosário Lima, 2018).

Hoje, a referida plataforma abrange 144 municípios paraenses e outros estados do Brasil, com cerca de 9.214 inscritos (ENEM PARÁ, 2021), de diferentes perfis socioeconômicos, culturais e regionais, esses observados através das diversas informações prestadas no cadastro inicial de acesso à mesma. Apresenta ainda, tradução da Língua Brasileira de Sinais (LIBRAS), possibilitando a inclusão de alunos surdos, favorecendo assim, a oportunidade e garantia do conhecimento oferecido pelo estado.

Todas as suas ferramentas tecnológicas aplicadas no processo de ensino, estão integradas com o Google for Education, uma solução construída com ferramentas colaborativas para gestores, professores e alunos, a fim de proporcionar engajamento e compartilhamento da aprendizagem (Bose, 2004). 
Nesse viés, torna-se relevante compreender o perfil dos usuários das plataformas on-line, através das informações pessoais cadastradas nas mesmas, uma vez que por meio desse perfil percebe-se as preferências de conteúdos, o comportamento social dos usuários (Almeida, et al., 2020), além de auxiliar no desenvolvimento de novos conteúdos personalizados.

Assim, alinhado à tendência do desenvolvimento, bem como do uso e aceitação cada vez mais frequente das plataformas on-line nos diferentes contextos, incluindo os serviços de e-governo aplicados à educação (Althonayan \& Althonayan, 2017), essa pesquisa busca compreender qual o perfil socioeconômico dos usuários que acessam a plataforma Enem Pará?

O objetivo deste trabalho é analisar o perfil socioeconômico dos usuários da plataforma Enem Pará, visto que através dessa análise é possível abranger os diferentes públicos que nela acessam, podendo resultar numa comunicação mais assertiva no desenvolvimento e compartilhamento de novos conteúdos da plataforma (Althonayan \& Althonayan, 2017; Costa Filho \& Branco, 2020).

Esse estudo se justifica na medida que é necessário a compreensão dos estágios iniciais do processo de implantação desse tipo de serviço para que se tenha maior desempenho e efetividade no atendimento dos usuários (Althonayan \& Althonayan, 2017).

Destarte, através do acesso e dados da plataforma Enem Pará, este trabalho apresenta resultados sobre o perfil socioeconômico dos usuários com base no uso metodológico de análise quantitativa, descritiva e análises cruzadas com o uso do software SPSS (Fávero \& Belfiore, 2017; Raupp \& Beuren, 2003).

Esse artigo está estruturado da seguinte forma: na próxima seção, será apresentado o referencial teórico que abrange conceitos de educação tecnológica, comportamento dos usuários de plataformas on-line e serviços de e-governo. Em seguida, é apresentada as bases metodológicas do trabalho e após, os resultados das análises dos dados. Finalmente, as considerações finais e implicações gerenciais.

\section{Referencial Teórico}

\subsection{Plataforma Enem Pará}

A plataforma Enem Pará, projeto de extensão da Universidade Federal do Pará (UFPA) criado no início do período pandêmico no estado do Pará, com vistas à democratização do conhecimento para alunos da rede pública de ensino que prestarão o Exame Nacional do Ensino Médio (Enem), foi financiado pelo Governo do Estado via Secretaria de Estado de Ciência, Tecnologia e Educação Tecnológica do Estado do Pará (Sectet), executado através do Parque de Ciência e Tecnologia, tem como principal objetivo contribuir para a transformação social alcançada por meio do ensino e a consequente inclusão de alunos da rede pública em cursos de nível superior, além de minimizar as difículdades de acesso à educação trazidas pela pandemia de Covid-19.

A plataforma foi desenvolvida em ambiente Web 3.0 e apresenta aulas gravadas com professores de todas as áreas do conhecimento abordadas no Enem. Todo o material é disponibilizado gratuitamente em um portal de conteúdos on-line (www.enempara.com), acessado a partir de login e senha disponibilizados a todos que se inscrevem. Trata-se de videoaulas exclusivas, além de material de apoio para leitura, simulados e exercícios, todos integrados com o Google for Education, uma solução construída com ferramentas colaborativas para gestores, professores e alunos, proporcionando o engajamento de pessoas a qualquer momento. Ademais, a referida plataforma exibe a tradução da Língua Brasileira de Sinais (Libras), possibilitando assim, a inclusão de alunos surdos.

Dessa forma, a plataforma entrou em operação no final do mês de junho de 2020 (Agência Pará, 2020) tendo 
algumas dificuldades de comunicação e informação aos potenciais usuários quanto ao serviço que estava sendo disponibilizado. Devido a isso, foram feitas divulgações em rádios, jornais, redes sociais e sites de prefeituras do estado, tudo para informar o público-alvo do serviço, que nesse caso são os estudantes aptos a realizar o Exame Nacional do Ensino Médio - ENEM.

Atualmente, a plataforma tem um pouco mais de 9.000 inscritos, na sua maioria oriundos da região metropolitana de Belém, porém conta com acessos de usuários de outros municípios do Pará e até de outros estados do Brasil. A mesma pretende um alcance e eficiência ainda maiores, de forma que com o passar do tempo, torne-se cada vez mais decisiva para o sucesso de milhares de alunos da rede pública de ensino frente aos desafios apresentados no processo de ensino aprendizagem.

Portanto, a utilização de recursos tecnológicos, a fim de democratizar o acesso à informação, ampliar e dinamizar a prestação de serviços públicos, é percebida como um grande potencial para a efetivação e eficiência na interação entre governo e sociedade (Sena Neto, 2021), bem como para a melhoria da qualidade do serviço público oferecido e integração de políticas públicas para a sociedade.

\subsection{Serviço público e E-Governo}

$\mathrm{O}$ modo com que as organizações se constituem e interagem atualmente com o mundo tem sido impactada de maneira singular, pelo fato de introduzir novas ferramentas tecnológicas baseadas na interatividade, informação, colaboração, revelando novos padrões comunicativos, onde por sua vez, as organizações públicas não ficam de fora (Gelatti, De Souza \& Da Silva, 2015).

Nesse sentido, as práticas adotadas pelo setor governamental estão relacionadas em grande parte ao conceito de governo eletrônico, ou e-gov, o qual faz uso estrategicamente das TDIC's nas relações do setor público com os cidadãos, usuários e demais participantes dessa relação com o Estado (Mesquita, 2019). Portanto, o e-gov não se restringe apenas à automação do serviço público, mas envolve uma mudança na maneira com que o governo, através do uso das tecnologias, atinge os seus objetivos, incluindo assim, melhoria nos serviços prestados, aumento da eficiência, melhor governança, elaboração e monitoramento de políticas públicas e aumento da transparência.

\subsection{Perfil dos usuários de plataformas tecnológicas}

A disponibilização e utilização de diferentes plataformas on-line tem se expandido no mundo todo, e consequentemente o número de usuários também (Silveira, et al., 2018). Nessa perspectiva, Costa Filho e Branco (2020) e Statista (2019) inferem em seus estudos que cerca de 2,62 bilhões de usuários acessaram diferentes plataformas durante o ano de 2018 e que a projeção para este ano de 2021 é de 3 bilhões.

Assim, os avanços e mudanças em rede proporcionam a intensificação e popularização das plataformas, além de possibilitar que os usuários dessas, sejam produtores de conteúdos e dados (Machado, 2018). Dessa forma, torna-se cada vez mais importante estudos acerca do perfil daqueles que acessam plataformas on-line disponíveis para as mais diversas utilidades, a fim de melhorar a interação entre usuário e plataforma, bem como o aperfeiçoamento do serviço disponibilizado.

Machado (2018) infere ainda, que plataformas de grande acesso como o Facebook possuem um saber e poder enorme sobre os usuários que nela interagem, visto as constantes análises de dados dos perfis desses, possibilitadas pelas tecnologias.

Há alguns estudos sobre o perfil de usuários de determinadas plataformas, como por exemplo os de Coelho et al., (2017), o qual analisa o perfil socioeconômico dos usuários da plataforma Uber, além de fatores relevantes que levam à utilização desta plataforma. No estudo, são apresentadas características pessoais de gênero, idade, atividade desempenhada e 
renda dos usuários, características essas importantes para elucidar o perfil daqueles que utilizam a referida plataforma de mobilidade.

Costa Filho e Branco (2020), por sua vez, analisaram o perfil dos usuários das principais plataformas de mídias sociais na Internet, formado por alunos entrantes de uma IES no Paraná. Nesse contexto, os autores puderam analisar que dentre as plataformas de mídia social mais utilizadas, o WhatsApp tem $98,7 \%$ de aceitação e utilização entre os entrevistados, e que plataformas de uso mais profissional como o Skype e Linkedin ainda são pouco utilizadas. Além disso, a pesquisa demonstrou que cerca de 36,7\% dos usuários das plataformas de mídias sociais buscam acesso para fins de estudos.

Por sua vez, Pereira et al., (2014), inferem sobre a extração de perfil e de contexto em redes sociais para recomendação de recursos educacionais, através de informações geradas espontaneamente, por meio do uso das redes sociais, explorando o tempo dispendido por estudantes nessas, auxiliando assim, no processo de seleção de conteúdos e recursos educacionais de acordo com o perfil de cada um, auxiliando na identificação de suas necessidades e interesses.

Castro e Adão (2015) realizaram uma análise acerca do perfil de usuários da internet, onde discorrem que a desigualdade social também se denota no meio digital, uma vez que segundo os autores dentre 10\% dos usuários mais pobres, apenas $0,6 \%$ têm acesso à internet, e entre os $10 \%$ dos usuários mais ricos 56,3\% conectam-se à internet. Além disso, as autoras ressaltam que os usuários declarados de raça branca acessam mais a internet do que os usuários declarados de raça negra. Nesse contexto, percebe-se um contraste, uma vez que o Brasil ocupa a $5^{a}$ posição no ranking dos países em população on-line (Brasil, 2021), porém a conectividade ainda não está ao alcance da grande parcela da população da base da pirâmide social. Castro e Adão (2015) perceberam ainda, que o perfil de faixa etária dos usuários que mais acessam a internet é de 16 a 24 anos e que o maior crescimento de usuários se deu entre aqueles com grau de instrução correspondente ao ensino médio.

No que concerne a pesquisas voltadas especificamente ao perfil de usuários de plataformas de conteúdos educacionais percebe-se uma escassez, no entanto, faz-se necessário compreender o desenvolvimento de plataformas on-line como forma de prover o ensino e a aprendizagem a partir da flexibilidade do formato, do tempo e espaço oferecido pelas tecnologias (Rieber, 2017). Nesse viés, este estudo busca contribuir para a compreensão do perfil socioeconômico dos indivíduos que acessam uma determinada plataforma de compartilhamento de conteúdos de aprendizagem, voltados inicialmente, para o acesso de estudantes do estado do Pará, mais especificamente àqueles que já concluíram o ensino médio ou que estão cursando o último ano e que prestarão o Exame Nacional do Ensino Médio.

A plataforma Enem Pará foi desenvolvida em meio a um cenário de dificuldades nos diferentes setores, seja ele econômico, social e educacional, a fim de propiciar o acesso à aprendizagem aos estudantes da rede pública de educação, os quais tiveram as dificuldades no processo de ensino acentuadas pela suspensão das aulas presenciais, e ainda com o intuito de minimizar as dificuldades educacionais e sociais, uma vez que essas tornam ainda mais precário o acesso desses estudantes do ensino médio à universidade.

Face ao cenário de disparidade de acesso à educação, Brazorotto (2017) evidencia que no seu contexto histórico, a educação é mediada por contradições, como por exemplo as desigualdades regionais e de renda, além disso, infere também que as desigualdades educacionais são menores entre as mulheres, pois estas apresentam maior ano médio de instrução quando comparadas aos homens.

Diante das análises e discussões acerca do perfil de usuários em diversos contextos, a internet através do desenvolvimento de ferramentas que corroborem o amplo acesso, busca contribuir para o desenvolvimento da qualidade de vida, bem como para o sentimento de inclusão de pessoas e seu senso de empoderamento como cidadãos. (Previte, Hearn \& Dann, 2001), empoderamento esse advindo da oportunidade de acesso à educação. Nesse contexto, Pedro et al. (2020) inferem que a oferta e o acesso da população de baixa renda ao ensino superior pode significar melhoria na qualidade de vida, 
sobretudo no que diz respeito ao aspecto financeiro, além de significar a inserção no mercado de trabalho, de forma mais qualificada e desenvolvendo habilidades e competências cognitivas mais complexas.

No que concerne ao uso da internet no contexto educacional, a educação on-line e EAD, as quais o usuário se conecta a uma plataforma virtual e encontra materiais, conteúdos e atividades, trazendo flexibilidade e desenvolvendo uma aprendizagem ativa e compartilhada tem sido predominante, uma vez das diversas possibilidades audiovisuais, interativas, de fácil acesso e abaixo ou nenhum custo (Moran, 2007).

Nesse cenário e ainda, diante de uma pandemia que traz inúmeras dificuldades, faz - se necessário o setor público intensificar a demanda quanto à eficiência e a qualidade na prestação de serviços, oferecendo maior acesso à informação e conhecimento, por meio dos avanços tecnológicos amplamente difundidos e experimentados no espaço virtual (Althonayan \& Althonayan, 2017). Portanto, percebe-se a necessidade de modificação na lógica que orienta a oferta e a prestação de serviços públicos, buscando focar-se mais na aproximação com o público atendido, bem como na satisfação do usuário (Ocde, 2015).

Aspectos como a adoção de tecnologias móveis sem fio tem sido amplamente adotada em serviços públicos, com vistas a atender de forma cada vez mais eficiente a população (Kushchu \& Kuscu, 2003). Isso exige um grande esforço de infraestrutura e treinamento dos servidores públicos e usuários (o cidadão) para que exista a efetividade na política de atendimento à sociedade (Bose, 2004).

Assim, a construção e efetivação da plataforma Enem Pará consiste numa prática do governo em potencializar a inclusão, facilitação do acesso à informação, a participação e o controle social da gestão pública a partir da disponibilização de canais de interação (Pinheiro, 2017).

\section{Metodologia}

Este trabalho tem quanto aos objetivos de pesquisa uma abordagem exploratória (Raupp \& Beuren, 2003), pois ainda há pouco conhecimento sobre quem são os usuários da plataforma Enem Pará e principalmente, se a mesma está cumprindo com os aspectos delineadores para sua criação, ou seja, se está atendendo principalmente os estudantes paraenses que irão fazer o exame nacional do ensino médio (ENEM).

Quanto aos procedimentos, essa pesquisa visa analisar um único caso (Raupp \& Beuren, 2003), pois a plataforma do Enem Pará reúne informações de 9.214 estudantes, onde o processo de cadastramento (acesso) requer que o usuário preencha uma quantidade relativamente significativa e detalhada de informações, como mostra a Figura 1. 
Figura 1 - Aba de cadastramento do Enem Pará

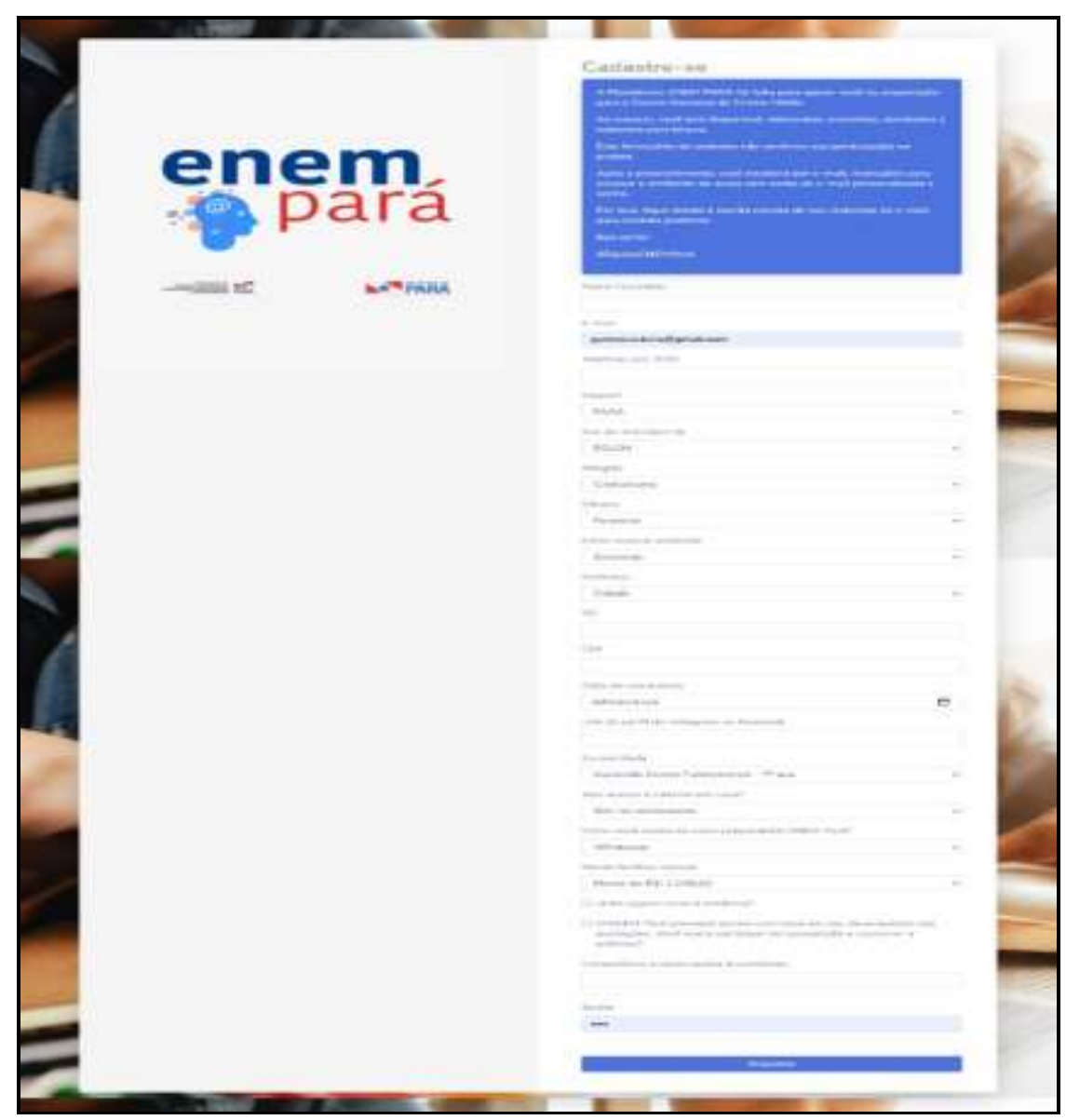

Fonte: Site Enem Pará (2020).

Quanto à abordagem do problema, essa pesquisa se caracteriza como quantitativa (Raupp \& Beuren, 2003), com o uso de dados secundários, pois os dados foram disponibilizados pelos gerenciadores da plataforma Enem Pará para o desenvolvimento do estudo. A apresentação dos resultados será por meio de estatística descritiva e análises cruzadas com o uso do software SPSS, com foco na verificação do perfil dos usuários (Fávero \& Belfiore, 2017).

\section{Resultados}

Como essa pesquisa tem por objetivo conhecer o perfil dos usuários da plataforma Enem Pará, foram criados gráficos e tabelas que tem por finalidade a demonstração de aspectos relevantes quanto às características socioeconômicas dos inscritos. Inicialmente foi verificado de quais cidades os usuários acessam a plataforma, nesse sentido observou-se o registro de 170 cidades diferentes, a grande maioria do estado do Pará, porém existe registro de municípios de outros estados da federação, como Macapá - AP, Imperatriz - MA e Belo Horizonte - MG. No entanto, a capital do estado do Pará (Belém) responde pelo maior número de usuários cadastrados na plataforma (30,5\%), seguido de Ananindeua-PA (8,3\%), Santarém - PA (5,9\%) e Abaetetuba-PA (4,2\%). Assim, a plataforma cumpre com o objetivo de atender os estudantes paraenses que representam $99,91 \%$ do total de inscritos.

Outro aspecto importante é quanto ao gênero feminino que representa $67,6 \%$, portanto a maioria dos inscritos. O gênero masculino, representa $31,7 \%$ e os outros $0,7 \%$ se declararam do grupo LGBTQIA+. Outro ponto de importante registro é que a maioria dos estudantes se declarou morador de zonas urbanas dos municípios (89\%). 
Quanto ao nível de escolaridade, 44,6\% informaram que terminaram o ensino médio e $40 \%$ que estavam terminando o último ano do ensino médio, dessa forma a plataforma tem cumprido com o objetivo de atender primordialmente os estudantes que estavam habilitados a concorrer por vagas nas Universidades via o ENEM. Contudo, 15,4\% dos estudantes declaram estar matriculados no $1^{\circ}$ ou $2^{\circ}$ ano do ensino médio $(13,3 \%)$ e no ensino fundamental $\left(7^{\circ}, 8^{\circ}\right.$ e $9^{\circ}$ anos) o restante $2,1 \%$.

Foram analisadas também informações quanto ao gosto musical e religião, onde a maioria dos usuários se declararam católicos $(39,84 \%)$ e gostar de música sertaneja (35,75\%). Porém, observa-se um número expressivo de estudantes que se declaram evangélicos $(33,63 \%)$ e têm como preferência o estilo musical gospel $(18,54 \%)$.

O processo de conhecimento da plataforma e dos serviços oferecidos por ela, também foram informados pelos inscritos. Nesse sentido a rede social Whatsapp foi predominante, 69,6\%, seguido do jornal impresso com 19,9\% e os sites das prefeituras com 7,4\%, por fim, 3,0\% dos usuários informaram ter tido conhecimento do serviço oferecido pelo governo do estado, via e-mail e rádio.

Foi solicitado pela plataforma informações quanto à experiência do usuário com a educação a distância - EAD, nesse sentido a maioria dos inscritos informou já ter dito contato com essa modalidade de ensino, sendo $79 \%$, contra $21 \%$ que disseram não ter dito aulas neste formato. Esses dados estão de acordo com o apresentado no Gráfico 1, que trata sobre o acesso dos inscritos à internet. A maioria informou ter acesso à rede mundial de computadores via celular.

Gráfico 1 - Acesso à internet.

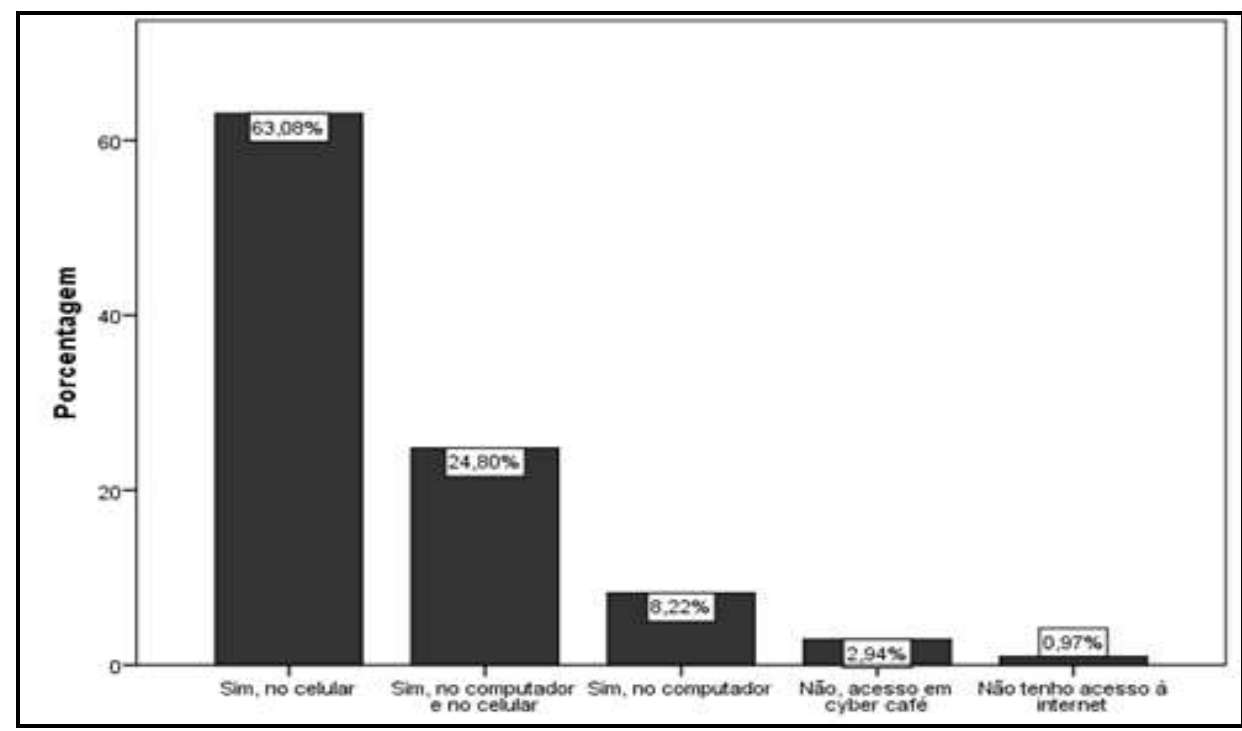

Fonte: Dados da pesquisa (2020).

Ainda considerando a análise do perfil dos inscritos na plataforma Enem Pará, foi verificada a distribuição por renda, ver Tabela 1. 
Tabela 1 - Distribuição por renda familiar.

\begin{tabular}{ccccc}
\hline & Frequência & Porcentagem & $\begin{array}{c}\text { Porcentagem } \\
\text { válida }\end{array}$ & $\begin{array}{c}\text { Porcentagem } \\
\text { cumulativa }\end{array}$ \\
\hline $\begin{array}{c}\text { Menor de R\$: } \\
1.039,00\end{array}$ & 5542 & 60,1 & 60,1 & 60,1 \\
$\begin{array}{c}\text { Entre R\$: } \\
1.039,00 \text { e R\$: } \\
\text { 2.078,00 } \\
\text { Entre R\$: }\end{array}$ & 2566 & 27,8 & 27,8 & 88 \\
$\begin{array}{c}2.078,00 \text { e R\$: } \\
\text { 4.156,00 }\end{array}$ & 758 & 8,2 & & \\
$\begin{array}{c}\text { Acima de R\$: } \\
\text { 4.156,00 }\end{array}$ & 348 & 3,8 & 3,2 & 100,2 \\
\hline Total & 9214 & 100 & 100 & \\
\hline
\end{tabular}

Fonte: Dados da pesquisa (2020).

Uma outra premissa para a implantação do serviço de aulas gratuitas online, pelo governo do estado do Pará, foi o atendimento aos estudantes de escolas públicas estaduais, que na grande maioria dos casos, tem o perfil de baixa renda (Castro \& Adão, 201; Neri, 2020). Nesse sentido, a análise cruzada de dados (Fávero \& Belfiore, 2017), pode contribuir para compreensão do grau de associação entre variáveis, com a finalidade de compreensão mais detalhada do perfil dos inscritos. Dessa forma, a Tabela 2 apresenta o cruzamento entre gênero e renda familiar. Mostra ainda, que do total de inscritos que se declararam com renda familiar inferior a $\mathrm{R} \$ 1.039,00$ (um mil e trinta e nove reais), 71,8\% são do sexo feminino. Além disso, a estatística $\chi^{2}=160,802, \mathrm{df}=15, \mathrm{p}$ - valor $<0,001$, demonstrando que as variáveis são dependentes entre si.

Tabela 2 - Tabulação cruzada renda familiar * gênero.

\begin{tabular}{|c|c|c|c|c|c|c|c|c|c|}
\hline & & & \multicolumn{6}{|c|}{ Gênero } & \multirow[t]{2}{*}{ Total } \\
\hline & & & Feminino & Homossexual & Lésbicas & Masculino & $\begin{array}{c}\text { Não } \\
\text { Listado }\end{array}$ & Trans & \\
\hline \multirow{8}{*}{$\begin{array}{l}\text { Renda } \\
\text { familiar }\end{array}$} & Acima de & Contagem & 183 & 1 & 0 & 164 & 0 & 0 & 348 \\
\hline & $\begin{array}{c}\mathrm{R} \$: \\
4.156,00\end{array}$ & $\begin{array}{c}\% \text { em } \\
\text { renda } \\
\text { familiar }\end{array}$ & $52,60 \%$ & $0,30 \%$ & $0,00 \%$ & $47,10 \%$ & $0,00 \%$ & $0,00 \%$ & $100,00 \%$ \\
\hline & Entre R\$: & Contagem & 1645 & 5 & 0 & 908 & 2 & 6 & 2566 \\
\hline & $\begin{array}{c}1.039,00 \\
\text { e R\$: } \\
2.078,00\end{array}$ & $\begin{array}{c}\% \text { em } \\
\text { renda } \\
\text { familiar }\end{array}$ & $64,10 \%$ & $0,20 \%$ & $0,00 \%$ & $35,40 \%$ & $0,10 \%$ & $0,20 \%$ & $100,00 \%$ \\
\hline & Entre R\$: & Contagem & 421 & 4 & 0 & 332 & 0 & 1 & 758 \\
\hline & $\begin{array}{c}2.078,00 \\
\text { e R\$: } \\
4.156,00\end{array}$ & $\begin{array}{c}\% \text { em } \\
\text { renda } \\
\text { familiar }\end{array}$ & $55,50 \%$ & $0,50 \%$ & $0,00 \%$ & $43,80 \%$ & $0,00 \%$ & $0,10 \%$ & $100,00 \%$ \\
\hline & Menor de & Contagem & 3979 & 28 & 3 & 1518 & 6 & 8 & 5542 \\
\hline & $\begin{array}{c}\mathrm{R} \$: \\
1.039,00\end{array}$ & $\begin{array}{l}\% \text { em } \\
\text { renda } \\
\text { familiar }\end{array}$ & $71,80 \%$ & $0,50 \%$ & $0,10 \%$ & $27,40 \%$ & $0,10 \%$ & $0,10 \%$ & $100,00 \%$ \\
\hline \multirow[b]{2}{*}{ Total } & & Contagem & 6228 & 38 & 3 & 2922 & 8 & 15 & 9214 \\
\hline & & $\begin{array}{c}\% \text { em } \\
\text { renda } \\
\text { familiar }\end{array}$ & $67,60 \%$ & $0,40 \%$ & $0,00 \%$ & $31,70 \%$ & $0,10 \%$ & $0,20 \%$ & $100,00 \%$ \\
\hline
\end{tabular}


Quando comparado a renda familiar com a experiência de ter estudado à distância, os percentuais apresentados mostram que em todas as faixas de renda o percentual de já ter dito a experiência com aulas em EAD é bem alto, mesmo entre os que declaram ter renda familiar inferior a $\mathrm{R} \$ 1.039,00$ (um mil e trinta e nove reais), sendo de 72,7\%. A estatística de Pearson apresentou $\chi^{2}=336,221, \mathrm{df}=3, \mathrm{p}$ - valor $<0,001$, demonstrando a dependência das variáveis.

Tabela 3 - Tabulação cruzada renda familiar * Já estudou a distância?

\begin{tabular}{|c|c|c|c|c|c|}
\hline & & & $\begin{array}{r}\text { Já est } \\
\text { distâ }\end{array}$ & $\begin{array}{l}\text { dou a } \\
\text { cia? }\end{array}$ & Total \\
\hline & & & NÃO & SIM & \\
\hline \multirow{8}{*}{$\begin{array}{l}\text { Renda } \\
\text { familiar }\end{array}$} & \multirow{2}{*}{$\begin{array}{c}\text { Acima de } \\
\mathrm{R} \$ 4.156,00\end{array}$} & Contagem & 39 & 309 & 348 \\
\hline & & $\begin{array}{c}\% \text { em renda } \\
\text { familiar }\end{array}$ & $11,20 \%$ & $88,80 \%$ & $100,00 \%$ \\
\hline & \multirow{2}{*}{$\begin{array}{c}\text { Entre R\$ } \\
1.039,00 \mathrm{e} \\
\mathrm{R} \$: \\
2.078,00\end{array}$} & Contagem & 283 & 2283 & 2566 \\
\hline & & $\begin{array}{l}\% \text { em renda } \\
\text { familiar }\end{array}$ & $11,00 \%$ & $89,00 \%$ & $100,00 \%$ \\
\hline & \multirow{2}{*}{$\begin{array}{c}\text { Entre R\$ } \\
2.078,00 \mathrm{e} \\
\mathrm{R} \$: \\
4.156,00\end{array}$} & Contagem & 99 & 659 & 758 \\
\hline & & $\begin{array}{l}\% \text { em renda } \\
\text { familiar }\end{array}$ & $13,10 \%$ & $86,90 \%$ & $100,00 \%$ \\
\hline & \multirow[b]{2}{*}{$\begin{array}{c}\text { Menor de } \\
\mathrm{R} \$ 1.039,00\end{array}$} & Contagem & 1514 & 4027 & 5541 \\
\hline & & $\begin{array}{l}\% \text { em renda } \\
\text { familiar }\end{array}$ & $27,30 \%$ & $72,70 \%$ & $100,00 \%$ \\
\hline \multirow[b]{2}{*}{ Total } & & Contagem & 1935 & 7278 & 9213 \\
\hline & & $\begin{array}{l}\% \text { em renda } \\
\text { familiar }\end{array}$ & $21,00 \%$ & $79,00 \%$ & $100,00 \%$ \\
\hline
\end{tabular}

Fonte: Dados da pesquisa (2020).

Corroborando com essa análise, o Gráfico 2 apresenta os dados cruzados da renda familiar (eixo x) e o acesso à internet (eixo y). Ainda os valores de $\chi^{2}=1276,031$, df $=12, \mathrm{p}$ - valor $<0,001$, demonstrando o alto grau de dependência entre as variáveis. Sendo que a grande maioria dos que se declararam com renda familiar inferior a $\mathrm{R} \$ 1.039,00$ (um mil e trinta e nove reais), informaram ter acesso à internet via celular. 
Gráfico 2 - Análise cruzada das variáveis renda familiar x Acesso à internet.

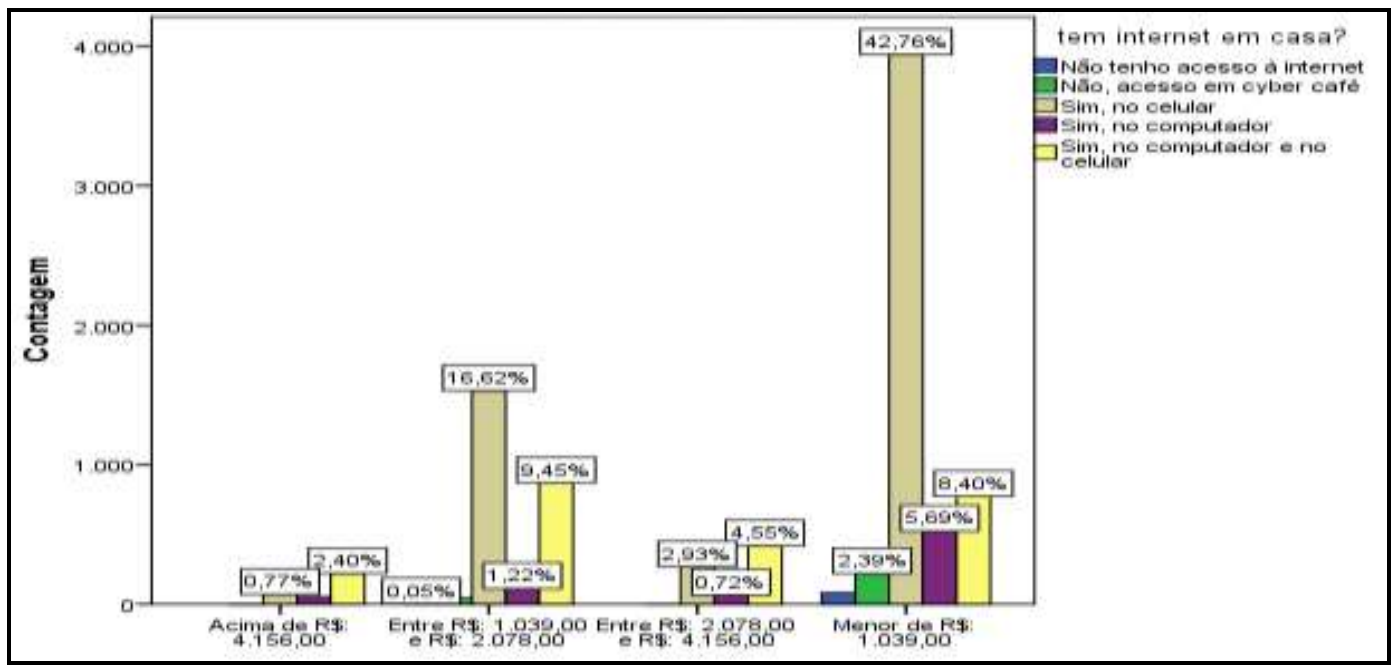

Fonte: Dados da pesquisa (2020).

Outra importante análise cruzada se refere a como os inscritos obtiveram informações quanto à existência do portal Enem Pará (comunicação do governo com o cidadão) e a renda familiar (ver Tabela 4). Embora o WhatsApp tenha sido a forma mais frequente de acesso à informação do serviço oferecido pelo governo, é entre os que declararam rendimento superior a $\mathrm{R} \$ 4.156,00$ que está o maior percentual de informação via essa rede social, correspondendo a 72,4\%. A dependência entre os construtos apresentou o $\chi^{2}=54,891, \mathrm{df}=12, \mathrm{p}$ - valor $<0,001$, demonstrando que o acesso à informação também depende da renda familiar.

Tabela 4 - Tabulação cruzada renda familiar x marketing.

\begin{tabular}{|c|c|c|c|c|c|c|c|c|}
\hline & & & \multicolumn{5}{|c|}{ marketing } & \multirow[t]{2}{*}{ Total } \\
\hline & & & E-mail & Jornal & Rádio & $\begin{array}{c}\text { Site } \\
\text { oficial da } \\
\text { prefeitura }\end{array}$ & Whatsapp & \\
\hline \multirow{8}{*}{$\begin{array}{l}\text { Renda } \\
\text { familiar }\end{array}$} & & Contagem & 6 & 67 & 7 & 16 & 252 & 348 \\
\hline & $\begin{array}{c}\mathrm{R} \$: \\
4.156,00\end{array}$ & $\begin{array}{c}\% \text { em } \\
\text { renda } \\
\text { familiar }\end{array}$ & $1,70 \%$ & $19,30 \%$ & $2,00 \%$ & $4,60 \%$ & $72,40 \%$ & $100,00 \%$ \\
\hline & Entre R\$: & Contagem & 26 & 607 & 34 & 216 & 1683 & 2566 \\
\hline & $\begin{array}{c}1.039,00 \\
\text { e R\$: } \\
2.078,00\end{array}$ & $\begin{array}{c}\% \text { em } \\
\text { renda } \\
\text { familiar }\end{array}$ & $1,00 \%$ & $23,70 \%$ & $1,30 \%$ & $8,40 \%$ & $65,60 \%$ & $100,00 \%$ \\
\hline & Entre R\$: & Contagem & 6 & 134 & 12 & 42 & 564 & 758 \\
\hline & $\begin{array}{c}2.078,00 \\
\text { e R\$: } \\
4.156,00\end{array}$ & $\begin{array}{l}\% \text { em } \\
\text { renda } \\
\text { familiar }\end{array}$ & $0,80 \%$ & $17,70 \%$ & $1,60 \%$ & $5,50 \%$ & $74,40 \%$ & $100,00 \%$ \\
\hline & Menor de & Contagem & 75 & 1030 & 116 & 408 & 3913 & 5542 \\
\hline & $\begin{array}{c}\mathrm{R} \$: \\
1.039,00\end{array}$ & $\begin{array}{c}\% \text { em } \\
\text { renda } \\
\text { familiar }\end{array}$ & $1,40 \%$ & $18,60 \%$ & $2,10 \%$ & $7,40 \%$ & $70,60 \%$ & $100,00 \%$ \\
\hline \multirow{2}{*}{\multicolumn{2}{|c|}{ Total }} & Contagem & 113 & 1838 & 169 & 682 & 6412 & 9214 \\
\hline & & $\begin{array}{l}\% \text { em } \\
\text { renda } \\
\text { familiar }\end{array}$ & $1,20 \%$ & $19,90 \%$ & $1,80 \%$ & $7,40 \%$ & $69,60 \%$ & $100,00 \%$ \\
\hline
\end{tabular}




\section{Conclusão}

A implantação do projeto Enem Pará, teve como premissa oferecer conteúdos voltados às provas do ENEM, para os alunos da rede pública dos anos finais do ensino médio do estado e para alunos que já concluíram o ensino médio. Sendo que todos os usuários inscritos na plataforma se declararam como alunos da educação pública do estado, embora não fosse exigido nenhum tipo de comprovação quanto a essa afirmação.

Ainda assim, os dados descritivos demonstram que a grande maioria dos 9214 usuários da plataforma se declarou morador do estado do Pará. Abrangendo praticamente todos os municípios do estado. Porém, segundo o site Qedu.org.br, o estado do Pará teve em 2020, cerca de 87.289 estudantes matriculados no terceiro ano do ensino médio, o que deixa claro que muitos alunos não tiveram conhecimento do serviço oferecido e/ou condições de acesso à plataforma.

O perfil quanto à renda familiar, que é um importante indicador do efeito da política pública na sociedade, apresentou que a grande maioria dos inscritos declarou ter renda inferior a $\mathrm{R} \$ 1.039,00$, sendo que a frequência acumulada até a renda de $\mathrm{R} \$ 4.156,00$, é de 96,2\%, esses resultados informam que a plataforma está atendo os estudantes de baixa renda (Neri, 2020). Entretanto, a maioria desses estudantes informa dificuldades quanto ao acesso à internet, pois 63,08\% dos usuários só conseguem ter acesso ao conteúdo via celular, demonstrando que a população mais carente tem limitações de uso de dispositivos tecnológicos, sendo esse aspecto uma barreira para o desenvolvimento desses estudantes (Castro \& Adão, 2015).

Mesmo assim, surpreende o número de estudantes que informaram já ter tido alguma experiência com aulas on-line, demonstrando que os usuários buscam se conectar para encontrar materiais, conteúdos e atividades, buscando flexibilizar os estudos e o desenvolvimento de uma aprendizagem ativa e compartilhada, aspectos esses de acordo com os pressupostos de Moran (2007).

A maioria dos inscritos se declarou do gênero feminino (67,6\%), o que demonstra concordância com os estudos de Brazorotto (2017), o qual afirma que as pessoas desse gênero apresentam maior interesse quanto às questões educacionais. Outro aspecto interessante é o gosto musical dos usuários da plataforma, pois o estado do Pará tem uma cultura musical rica e diversa, com ritmos regionais bem marcantes e conhecidos nacionalmente, como o Brega, o Melody, o Carimbó e o Tecno Melody, onde esses ritmos obtiveram apenas 4,7\% da preferência.

Contudo, essas análises demonstram a essencialidade de verificar a efetividade do serviço público em seu estágio inicial para que a comunicação seja mais eficiente e as estratégias de oferecimento do serviço realmente atendam o públicoalvo (Althonayan \& Althonayan, 2017). Serviços com o uso de plataformas na web, especialmente no que tange a educação pública no estado do Pará, ainda são pouco usados, o que corrobora para compreensão da necessidade de diagnósticos permanentes quanto ao atendimento dos cidadãos (Kushchu \& Kuscu, 2003).

As análises cruzadas apresentaram o grau de dependência das relações entre as variáveis socioeconômicas observadas, essas análises tiveram como objetivo demonstrar que o perfil dos inscritos na plataforma justifica a continuidade do serviço oferecido, já que todos os relacionamentos apontam a dependência do acesso à informação (comunicação) e estudo, à renda familiar. Esses aspectos podem contribuir com a ampliação do serviço, como por exemplo, a adaptação da plataforma para o celular, buscando a ampliar a parceria com as prefeituras dos municípios paraenses, para que os munícipes possam ter maior conhecimento do serviço oferecido; outro ponto, é o uso das redes sociais, como o Facebook e Instagram, que podem contribuir para que um número maior de estudantes possa ter acesso à informação dos serviços oferecidos na plataforma.

Ainda foi possível observar que o formulário de inscrição, que permite acesso aos serviços oferecidos na plataforma, não contempla a declaração de raça, ponto importante na definição de cotas nas universidades públicas do país, sendo este um aspecto relevante para política pública de inclusão digital. Mais ainda, não contempla informações sobre a modalidade de serviço educacional, como por exemplo, se o estudante foi ou é aluno da educação de jovens e adultos (EJA) ou da educação 
Research, Society and Development, v. 11, n. 1, e10311124526, 2022

(CC BY 4.0) | ISSN 2525-3409 | DOI: http://dx.doi.org/10.33448/rsd-v11i1.24526

técnica/tecnológica, que são oferecidos pelo governo. Essas informações poderiam contribuir mais ainda com a assertividade das propostas de serviços oferecidos dentro da plataforma Enem Pará, bem como, com outras plataformas que venham a ser implantadas pelo governo do estado.

As limitações deste trabalho estão principalmente relacionadas ao método, pois uma análise descritiva, com questões qualitativas, pode lançar luz sobre algumas características dos usuários, que pode dar um indicativo do público-alvo do serviço, porém, pouco pode informar sobre a efetividade e a satisfação dos usuários.

Por fim, este trabalho busca contribuir com as estratégias de comunicação e inclusão digital de mais estudantes paraenses na plataforma Enem Pará, entendendo que esse é um importante serviço que o governo do estado tem oferecido para aqueles que irão prestar o exame nacional do ensino médio.

\section{Referências}

Agência Pará. (2021). Plataforma EnemPará registra quase 10 mil visitas em apenas 15 dias. https://agenciapara.com.br/noticia/20883/

Alexandroni, L. A., \& Arruda Filho, E. J. M. (2017, October). Valores Motivacionais do Consumo Automobilístico Brasileiro Analisados Por Meio de Uma Pesquisa Netnográfica no Contexto do Big Data. In CLAV 2017.

Almeida, G. R., Guimarães, I., Jacob Jr, A. F., \& Lobato, F. M. (2020, June). Fontes de dados gerados por usuários: quais plataformas considerar?. In Anais do IX Brazilian Workshop on Social Network Analysis and Mining (pp. 25-36). SBC.

Althonayan, M., \& Althonayan, A. (2017). E-government system evaluation: The case of users' performance using ERP systems in higher education. Transforming Government: People, Process and Policy. 11(3), 306-342.

Bose, R. (2004). E-government: infrastructure and technologies for education and training. Electronic Government, an International Journal, 1(4), 349-361.

Brasil. (2021b). Brasil está entre os cinco países do mundo que mais usam internet. Governo do Brasil. https://Www.Gov.Br/Pt-Br/Noticias/Transito-ETransportes/2021/04/Brasil-Esta-Entre-Os-Cinco-Paises-Do-Mundo-Que-Mais-Usam-

Internet\#: :Text=Brasi1\%20est $\%$ C3\%A1\%20entre $\% 20$ os $\% 20$ cinco $\% 20$ pa $\%$ C3\%Adses $\% 20$ do $\% 20$ mundo $\% 20$ que $\% 20 \mathrm{mais} \% 20$ usam $\% 20$ internet,

Pa\%C3\%Ads\%20participou\%20de\&Text=Com\%2078\%2c3\%25\%20de\%20brasileiros,No\%20uso\%20di\%C3\%A1rio\%20de\%20internet.

Brazorotto, C. M. (2017). Quem são os estudantes do ensino médio profissionalizante no Brasil e na Alemanha. Colóquio Nacional, 4.

Costa Filho, B. A., \& Branco, R. B. C (2020). Segmentação baseada no comportamento de uso de plataformas de mídias sociais por alunos entrantes de uma instituição de ensino superior e perfil dos usuários. XLIV Encontro da Anpad - EnANPAD.

Silveira, S. A., de Souza, J. A., Machado, D. F., \& Santos, C. O. (2018). Análise das plataformas de compartilhamento online e de suas práticas colaborativas. Revista Eletrônica Internacional de Economia Política da Informação, da Comunicação e da Cultura, 20(2), 7-23.

Enem Pará. (2021). Processo Seletivo. http://enempara.net.br.

Fávero, L. P., \& Belfiore, P. (2017). Manual de análise de dados: estatística e modelagem multivariada com Excel®, SPSS® e Stata®. Elsevier Brasil.

Gelatti, A. R., de Souza, R. L., \& da Silva, R. L. (2015). Poder Público, TIC e E-GOV: uma análise acerca do uso das novas tecnologias na administração pública. In Congresso Internacional De Direito E Contemporaneidade, $3^{\circ}$., Congresso Iberoamericano De Investigdores E Docentes De Direito E Informática.

Kushchu, I., \& Kuscu, H. (2003, July). From E-government to M-government: Facing the Inevitable. In the 3rd European Conference on e-Government (pp. 253-260). MCIL Trinity College Dublin Ireland.

Machado, D. (2018). A modulação de comportamento nas plataformas de mídias sociais. J. Souza., R. Avelino., \& S. Amadeu da Silveira, A sociedade de controle: Manipulação e modulação nas redes digitais, 47-69.

Martins, C., Giraffa, L. M. M., \& do Rosário Lima, V. M. (2018). Gamificação e seus potenciais como estratégia pedagógica no ensino superior. Renote, $16(1)$.

Mesquita, K. (2020). A evolução do governo eletrônico no Brasil e a contribuição das TIC na redefinição das relações entre governo e sociedade. Comunicologia-Revista de Comunicação da Universidade Católica de Brasília, Brasília, 12(2), 174-195.

Moran, J. M. (2007). Os modelos educacionais na aprendizagem on-line. São Paulo: USP.

Neri, M. (2020). Qual foi o impacto imediato da pandemia do Covid sobre as classes econômicas brasileiras?. FGV Social.

Ocde - Organização Para A Cooperação e Desenvolvimento Econômico. (2015). Recommendation of the council on digital government strategies. https://goo.gl/GQ3wi8. 
Research, Society and Development, v. 11, n. 1, e10311124526, 2022

(CC BY 4.0) | ISSN 2525-3409 | DOI: http://dx.doi.org/10.33448/rsd-v11i1.24526

Parada, A. R., dos Santos Portal, V. M., Rodrigues, M. D. A. T., \& Borba, E. Z. (2020). O uso de metodologias ativas no ensino remoto com alunos de uma IES durante a pandemia do Covid-19. Redin-Revista Educacional Interdisciplinar, 9(1).

Pedro, E. D. M., Leitão, J., \& Alves, H. (2020). Bridging intellectual capital, sustainable development and quality of life in higher education institutions. Sustainability, 12(2), 479 .

Pinheiro, B. (2017). Plataformas de democracia eletrônica: um retrato para gestores públicos. In X Congresso Consad de gestão pública.

Previte, J., Hearn, G., \& Dann, S. (2001). Profiling Internet Users' Participation in Social Change Agendas: An application of Q methodology. arXiv preprint cs/0109058.

Raupp, F. M., \& Beuren, I. M. (2006). Metodologia da pesquisa aplicável às ciências. Como elaborar trabalhos monográficos em contabilidade: teoria e prática. São Paulo: Atlas, 76-97.

Rieber, L. P. (2017). Participation patterns in a massive open online course (MOOC) about statistics. British Journal of Educational Technology, 48(6), 12951304.

Sena Neto, B. (2021). Governo eletrônico (e-Gov).

Soares, D. M. R., Teles, G., Sena, T. B. Q. L., Loureiro, R. C., \& De Lima, L. (2018). As Tecnologias Digitais da Informação e Comunicação (Tdics) na Prática Docente. Ciet: EnPED.

Statista. (2021). Number of social network users worldwide from 2010 to 2020. https://www.statista.com/statistics/278414/number-of-worldwide-socialnetworkusers 